Audiology

Neurotology
Audiol Neurotol 2013;18:307-316

DOI: $10.1159 / 000353534$
Received: January 8, 2013

Accepted after revision: June 4, 2013 Published online: September 5, 2013

\title{
Influence of Cochleostomy and Cochlear Implant Insertion on Drug Gradients following Intratympanic Application in Guinea Pigs
}

\author{
E.B. King ${ }^{a, b} \quad$ J.J. Hartsock ${ }^{c}$ S.J. O'Leary ${ }^{a} \quad$ A.N. Salt ${ }^{c}$ \\ a Department Otolaryngology, University of Melbourne, and ${ }^{\mathrm{b}}$ Bionics Institute, Melbourne, Vic., Australia; \\ 'Washington University School of Medicine, St Louis, Mo., USA
}

\section{Key Words}

Cochleostomy · Perforation - Cochlear implant - Electrode .

Pharmacokinetics · Drug distribution · Computational modeling

\begin{abstract}
Locally applied drugs can protect residual hearing following cochlear implantation. The influence of cochlear implantation on drug levels in the scala tympani (ST) after round window application was investigated in guinea pigs using the marker trimethylphenylammonium (TMPA) measured in real time with TMPA-selective microelectrodes. TMPA concentration in the upper basal turn of the ST rapidly increased during implantation and then declined due to cerebrospinal fluid entering the ST at the cochlear aqueduct and exiting at the cochleostomy. The TMPA increase was found to be caused by the cochleostomy drilling if the burr tip partially entered the ST.TMPA distribution in the second turn was less affected by implantation procedures. These findings show that basal turn drug levels may be changed during implantation and the changes may need to be considered in the interpretation of therapeutic effects of drugs in conjunction with implantation.

Copyright $\odot 2013$ S. Karger AG, Basel
\end{abstract}

\section{Introduction}

Hearing outcomes, especially with respect to music appreciation and speech perception in the presence of background noise, can be improved following cochlear implantation (CI) when residual hearing is protected [von Ilberg et al., 1999; Gantz and Turner, 2003; Gantz et al., 2005; Gstoettner et al., 2008]. Protection strategies are becoming increasingly important as patient selection for $\mathrm{CI}$ is widened to include patients with more residual hearing. In addition to soft surgical (SS) techniques when performing the cochleostomy [Lehnhardt, 1993; Kiefer et al., 2004; Eshraghi, 2006] and atraumatic electrode design [Adunka et al., 2004; Gantz and Turner, 2004; Lenarz et al., 2006; Baumgartner et al., 2007], residual hearing can be protected with pharmaceuticals such as glucocorticoid steroids following cochlear implant surgery [James et al., 2008; Chang et al., 2009]. Steroids can be administered intratympanically on the round window membrane (RWM) prior to the cochleostomy to inhibit an inflammatory response to the implant, which has resulted in improved cochlear function in humans [Jayawardena et al., 2012] and animals [Kiefer et al., 2004; Kiefer et al., 2007; Ye et al., 2007; James et al., 2008] along with a lowered incidence of dizziness in humans [Enticott et al., 2011]. A study in which function was assessed in non-implanted and implanted groups had

\section{KARGER}

E-Mail karger@karger.com

www.karger.com/aud (c) 2013 S. Karger AG, Basel

1420-3030/13/0185-0307\$38.00/0
Elisha King

Bionics Institute

384-388 Albert Street

East Melbourne, VIC 3002 (Australia)

E-Mail Eking@bionicsinstitute.org 
previously suggested that inner ear surgery could have a significant effect on intracochlear drug distribution [Eastwood et al., 2010]. In order to understand the best way to apply steroids locally to optimize protection, it is necessary to establish which aspects of the implantation procedure influence drug distribution.

Following intratympanic administration, the drug enters the inner ear through both the RWM and in the vicinity of the stapes footplate [King et al., 2011; Salt et al., 2012b]. In the sealed cochlea, substances predominantly move in the fluids by passive diffusion driven by concentration gradients. For short-term applications, the influence of longitudinal perilymph flow has been shown to be low [Ohyama et al., 1988; Salt and Ma, 2001]. When a guinea pig cochlea is perforated, however, fluid is expelled from the cochleostomy site since cochlear fluidic pressure exceeds atmospheric pressure. This causes an influx of cerebrospinal fluid (CSF) through the cochlear aqueduct (CA), inducing a bulk longitudinal flow between the CA and the cochleostomy. The magnitude of the flow rate is proportionate to the pressure differential across the perforation site. An apical flow of $1.0 \mu \mathrm{l} / \mathrm{min}$ has previously been measured following an apical perforation in a guinea pig [Ohyama et al., 1988]. Thus, bulk longitudinal flow plays a more substantial role in drug distribution when the cochlea is perforated.

The traditional method of performing a cochleostomy for $\mathrm{CI}$ is to use an otological drill and diamond burr to perforate the otic capsule anterior/inferior to the RWM to access the scala tympani (ST). However, studies have shown that direct drilling and suction can cause acoustic trauma resulting in the loss of residual hearing, particularly at low frequencies [Kylén and Arlinger, 1976; Doménech et al., 1989; Pau et al., 2007], and there is some concern that this route for CI may potentially cause damage to the spiral ligament and basilar membrane [Lehnhardt, 1993; Briggs et al., 2005]. This has led to the introduction of SS techniques [Lehnhardt, 1993; Kiefer et al., 2004; Eshraghi, 2006]. An SS technique to open the ST is to use a drill to expose the endosteum without perforating it, flushing away bone dust with Ringer's solution, and carefully incising the endosteum with a microlancet knife or needle immediately before implantation, whilst avoiding direct suction in the cochleostomy [Kiefer et al., 2004]. Another SS method gaining clinical interest is to insert the implant directly through the RWM, avoiding drilling of the otic capsule altogether to minimize the potential risk of damage to the delicate intracochlear structures [Skarzynski et al., 2007; Gudis et al., 2012]. Trials are also underway in vitro to perform the cochleostomy with smart robotic microdrills [Coulson et al., 2008] or with handheld $\mathrm{CO}_{2}$ lasers [Fishman et al., 2010; Cipolla et al., 2012].

We have previously shown that CI does not significantly influence the distribution of the marker gadolinium within the cochlea at $1 \mathrm{~h}$ [King et al., 2011], but the effect of the cochleostomy on drug distribution has not been previously quantified. This is an important consideration, however, in the event that the cochlea has been 'preloaded' with drug, as would occur following pre-implantation round or oval window drug delivery. The method performing the cochleostomy could potentially alter the intracochlear drug distribution through either fluid leakage or mixing of fluids, the nature of which must be understood if the correct drug dosage is to be delivered to the target structures in order to adequately protect residual hearing following CI. Furthermore, we explore the effects of implant insertion in greater detail with direct measures to verify our previous finding that implantation does not substantially redistribute intracochlear drug. This study investigated marker distribution in the ST basal turn (ST1) during these events in real time using an ion-selective microelectrode sealed into the otic capsule of the ST.

\section{Materials and Methods}

\section{Ion-Selective Microelectrode Experiments}

\section{Animal Preparation}

The study was conducted in accordance with the policies and recommendations of the United States Department of Agriculture and the National Institutes of Health $(\mathrm{NIH})$ guidelines for the handling and use of laboratory animals, and received approval from the Institutional Animal Care and Use Committee of Washington University under protocols 20070147 and 20100135.

A total of 10 pigmented $\mathrm{NIH}$-strain guinea pigs weighing 450 $600 \mathrm{~g}$, anaesthetized with $100 \mathrm{mg} / \mathrm{kg}$ sodium thiobutabarbital (Inactin; Sigma, St. Louis, Mo., USA) were used in the study. Anesthesia was maintained with $0.8-1.2 \%$ isoflurane in oxygen and the animals were ventilated via a tracheal cannula during surgery with tidal volume set to maintain end-tidal $\mathrm{CO}_{2}$ near $38 \mathrm{~mm} \mathrm{Hg} \mathrm{(5 \% ).} \mathrm{Heart} \mathrm{rate}$ and blood oxygen saturation were monitored continuously by Surgivet pulse oximeter (Waukesha, Wisc., USA). Rectal temperature was maintained at $39^{\circ} \mathrm{C}$ with a thermistor-controlled DC-powered heating blanket. The animals were mounted securely in a head holder and the right cochlea exposed by the ventrolateral approach. All experiments were performed as non-recovery procedures.

\section{Cochleostomy and Cochlear Implants}

The cochleostomy was formed in the basal turn of the guinea pig ST using either SS techniques $(n=3)$ or by using a dental drill fitted with a 0.75 -mm-diameter diamond cutting burr (Sunshine Diamonds; Dr. Hopf GmbH \& Co. KG, H001009; $\mathrm{n}=5$ ) or a 0.52-mm-diameter carbide burr (Sunshine Carbide; Dr. Hopf $\mathrm{GmbH} \& \mathrm{Co} . \mathrm{KG}, \mathrm{C} 1104005 ; \mathrm{n}=2$ ). The SS technique entailed 
thinning the bone initially with a dental burr and using a fine pick to perforate the otic capsule and remove bone fragments. In all cases, the cochleostomy fenestra was made approximately $1.5 \mathrm{~mm}$ from the lip of the RWM.

The cochlear implants used in the study were $100 \%$ biocompatible dummy electrodes with three platinum rings (sourced from the Department of Otolaryngology, University of Melbourne). They were cylindrical in shape with $0.4 \mathrm{~mm}$ diameter and were inserted $2.12 \mathrm{~mm}$ (to the edge of the third ring) into the basal turn of the ST through the cochleostomy.

\section{Ion-Selective Microelectrodes}

Ion-selective microelectrodes were sealed in the upper basal turn $(n=9)$ or second turn of the ST $(n=1)$ to monitor the perilymph concentration of the marker drug trimethylphenylammonium (TMPA) in real time. The position of the basal turn microelectrode was approximately $3.1 \mathrm{~mm}$ from the RWM and the second turn microelectrode was approximately $7.5 \mathrm{~mm}$ from the RWM when measured along the scala. The microelectrodes consisted of a double-barreled glass pipette (one barrel with internal filling fiber and one without). After the glass pipettes were pulled, they were stored in a humidity cabinet at $40^{\circ} \mathrm{C}$ and $70 \%$ humidity overnight. The barrel without fiber was silanized by exposure to dimethyldichlorosilane vapor (Sigma) followed by baking at $140^{\circ} \mathrm{C}$ for $1 \mathrm{~h}$. The tips were beveled to a diameter of $3-4 \mu \mathrm{m}$. The silanized ion barrel was filled with $500 \mathrm{mM} \mathrm{KCl}$ and the reference barrel was filled with $500 \mathrm{mM} \mathrm{NaCl}$. TMPA-selective ion exchanger was made from $5 \%$ potassium tetrakis (4-chlorophenyl) borate in 2-nitrophenyloctylether (Fluka/Sigma, N.Y., USA) that was preequilibrated by storage in contact with concentrated TMPA solution. A short column of ion exchanger was drawn into the tip of the ion barrel by suction. The microelectrodes were connected to a high-impedance electrometer through $\mathrm{Ag} / \mathrm{AgCl}$ wires. Potentials were recorded differentially between the ion and reference barrels of the electrode. Each microelectrode was individually calibrated before use in a series of 5 standards containing 0,20,200, 2,000 and $20,000 \mu \mathrm{M}$ in a background of artificial perilymph, held at $39^{\circ} \mathrm{C}$ in a water circulation chamber. TMPA measurements from the animal were collected under computer control, with 10 measurements averaged and stored at 5-second intervals.

\section{Experimental Procedure}

The auditory bulla was accessed using a ventrolateral approach and opened to expose the cochlea. TMPA-selective microelectrodes were sealed into either the upper basal turn $(n=9)$ or second turn $(\mathrm{n}=1)$ of the ST using methods detailed elsewhere [Ohyama et al., 1988]. Briefly, after the mucosa was removed, the bony wall was thinned with a flap knife. Any fluid present was dried with paper wicks, and the bone was allowed to air-dry and was then coated with a thin layer of cyanoacrylate glue. A thin layer of 2-part silicone adhesive was applied over the cyanoacrylate glue to make the surface hydrophobic, and a small fenestra (approximately 30-40 $\mu \mathrm{m}$ ) was made through the adhesives and the bone using a fine pick $(0.3-\mathrm{mm}$, $30^{\circ}$ Storz N1705 80; Bausch and Lomb Inc.). The microelectrode was then positioned in the fenestra and sealed in place with a droplet of cyanoacrylate glue. This method allows the microelectrode to be placed with no subsequent perilymph leakage at the insertion site.

An artificial perilymph solution $(127.5 \mathrm{mM} \mathrm{NaCl}, 3.5 \mathrm{mM} \mathrm{KCl}$, $25 \mathrm{mM} \mathrm{NaHCO}_{3}, 1.2 \mathrm{mM} \mathrm{MgCl}_{2}, 0.75 \mathrm{mM} \mathrm{NaH}_{2} \mathrm{PO}_{4}, 1.3 \mathrm{mM}$ $\mathrm{CaCl}_{2}, 11 \mathrm{mM}$ glucose) containing $20 \mathrm{mM}$ TMPA was delivered

Influence of Cochleostomy and Cochlear Implant Insertion on Drug Gradients continuously to the RW niche at a rate of $5 \mu \mathrm{l} / \mathrm{min}$ for approximately $30 \mathrm{~min}$ prior to cochleostomy and implantation. Paper wicks were used to prevent fluid accumulation in the middle ear space. This procedure established a gradient for TMPA along the ST with highest levels at the basal entry site near the RWM. The cochleostomy was formed in the basal turn of the ST using a drill or SS techniques, and the ST was implanted with a cochlear implant electrode array.

In some experiments, the timing of events was recorded on a digital audio recording made with Audacity (http://audacity. sourceforge.net/), which was time-synchronized with the automated TMPA measurements.

Computer simulations of the experimental protocol were performed with version 3.081 of the Washington University Cochlear Fluids Simulator (available for download from our website at http:// oto.wustl.edu/cochlea/). Parameters for TMPA distribution are incorporated into the program, based on a variety of experimental protocols involving in situ TMPA measurements and measurements of TMPA concentrations in perilymph samples [Salt et al., 2012a].

\section{In vitro Experiments}

In order to visualize the influence of the experimental procedures on fluid movements and substance distribution, we set up a number of in vitro experiments using solutions marked with fluorescein. In one, a cochlear implant electrode was inserted into a 1.0-mm-diameter closed-ended, blunt tip glass capillary tube $(5 \mu \mathrm{l}$, VWR International, Radnor, Pa., USA) filled with lactated Ringer's solution. Before the insertion, the fluid region near the tube opening was injected with solution in which $20 \mathrm{mM} \mathrm{NaCl}$ was replaced with sodium fluorescein (Sigma-Aldrich, Taufkirchen, Germany). The electrode dummy was inserted into the tube through the Ringer's solution/fluorescein interface using a micromanipulator during which the distribution of fluorescein was recorded with videography.

In some experiments, we used a tube filled with lactated Ringer's solution which had an opening made in the side wall and fluorescein solution injected at one side of the opening. The tip of the dental burr was inserted into the tube and the change of fluorescein distribution was recorded with videography when the burr was activated. In other experiments, a stream of fluorescein solution was injected from a micropipette in a beaker of lactated Ringer's solution. The influence of dental burrs and implant electrode movements on fluorescein distribution was observed. This allowed us to compare induced changes of distribution under conditions where there were no nearby walls or boundaries.

In all of these experiments, the solutions used were simple electrolyte solutions (lactated Ringer's solution or fluid containing fluorescein) with solution viscosity expected to be close to that of perilymph.

\section{Results}

These experiments used TMPA as a marker to represent the distribution of drug applied locally to the RWM approximately $30 \mathrm{~min}$ before implantation. TMPA con- 


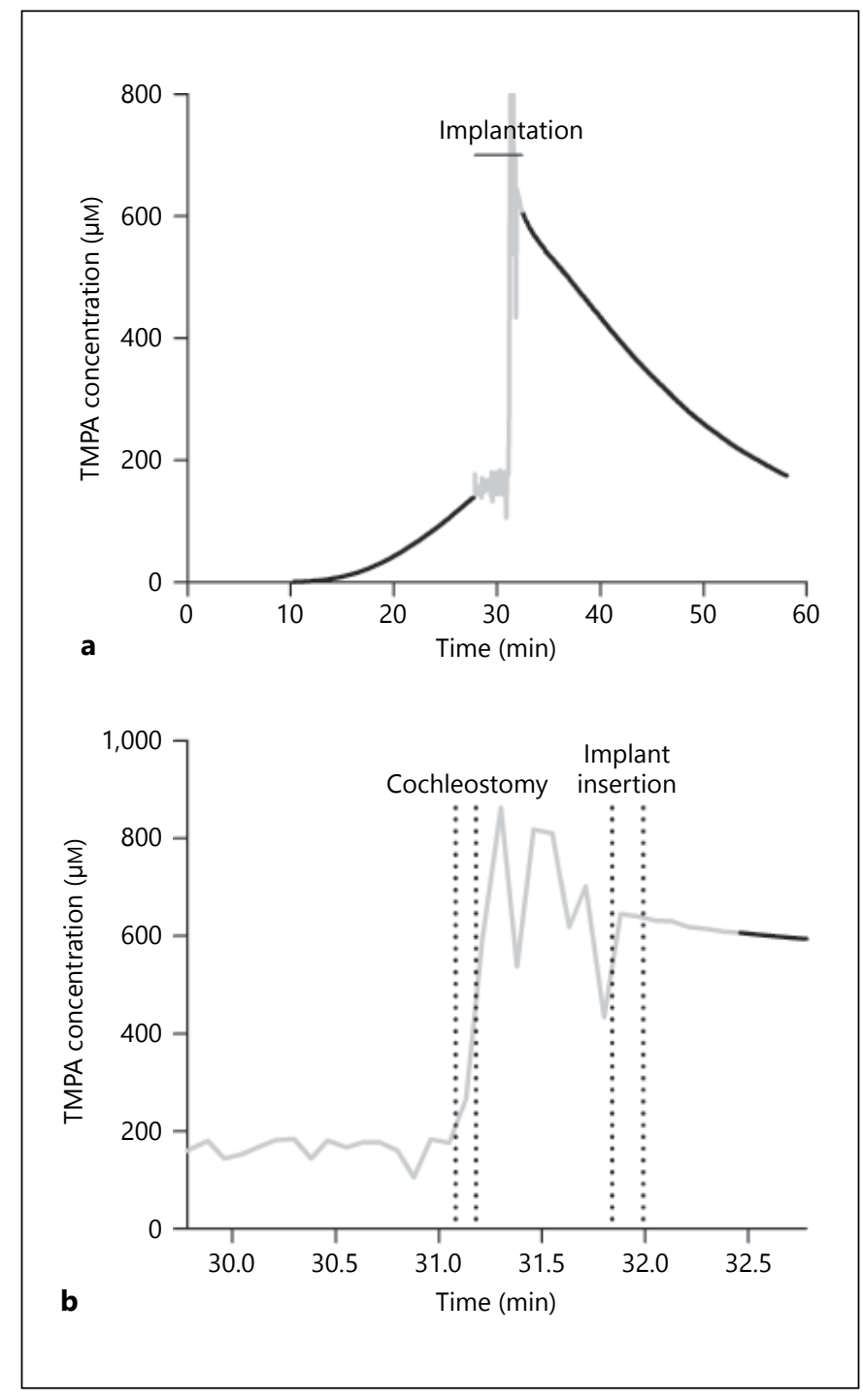

Fig. 1. a TMPA time course recorded from the basal turn of the ST following RW irrigation with $20 \mathrm{mM}$ TMPA commencing at zero time, followed by implantation, indicated by the gray, noisy area. The gray, noisy region was when a cochleostomy was drilled and an implant inserted into the ST. The noise results from electrical pickup by the high impedance microelectrode due to nearby motion and does not represent actual TMPA concentration change. TMPA irrigation was terminated at the time of implantation, but TMPA solution remained in the RW niche. Following implant insertion the measured TMPA became substantially higher but then declined progressively with time. b TMPA concentration during the implantation procedure shown with expanded time scale and with exact event timings derived from audio recordings of the procedure. The lines indicate the start and end of each procedure. Even though the trace is noisy due to movements near the microelectrode, it is apparent that the large increase of concentration is associated with making the cochleostomy rather than the insertion of the cochlear implant electrode. centration can be measured continuously with time throughout a procedure with an ion-selective microelectrode at a fixed location. TMPA time course curves are readily interpreted with computer models to give a realistic representation of how substances are distributed with distance in the ear as a function of time. The experiment in the upper panel of figure 1 shows the measured time course of TMPA in the upper basal turn of the ST before and after CI. At zero time, TMPA solution was irrigated across the RWM. After a delay of approximately $10 \mathrm{~min}$, during which TMPA diffuses from the RW region to the measurement site, TMPA concentration shows a progressive increase. Throughout the preimplantation period, there is a longitudinal gradient of TMPA along the ST, with the highest concentration near the RWM that progressively spreads further along the ST with time. During implantation (shown as gray in fig. 1) the measured concentration becomes noisy and unreliable due to movements of the experimenter near the highimpedance microelectrode introducing artifactual potentials. Immediately after implantation, when the trace becomes stable again, the measured TMPA level was substantially higher than would be expected by extrapolating the curve prior to implantation. This suggested that the implantation procedure in some manner affected the distribution of TMPA in the cochlea, increasing the TMPA level at the measurement location, which was just apical to the tip of the inserted implant. In order to carefully document the timing of different parts of the implantation procedure, we made digital audio recordings time-synchronized with the TMPA measurements. The audio recordings were used to distinguish the times when the cochleostomy was drilled and when the cochlear implant electrode was inserted, as shown in figure 1b. Although the measurements are noisy due to the experimental manipulations near the recording microelectrode, it was apparent that the TMPA concentration increased markedly when the cochleostomy was made rather than at the time of implant insertion. This experiment provided initial evidence against the cochlear implant electrode insertion being the cause of TMPA increase.

Figure 2 shows an experiment in which the different elements of the implantation procedure were further separated in time to allow undisturbed periods of TMPA recording. Digital audio recordings were again used to keep an accurate record of events with time. In this experiment, the cochleostomy was drilled carefully, pausing the procedure as soon as the ST was perforated and fluid appeared. At that time (fig. 2, initial drilling), the progres- 


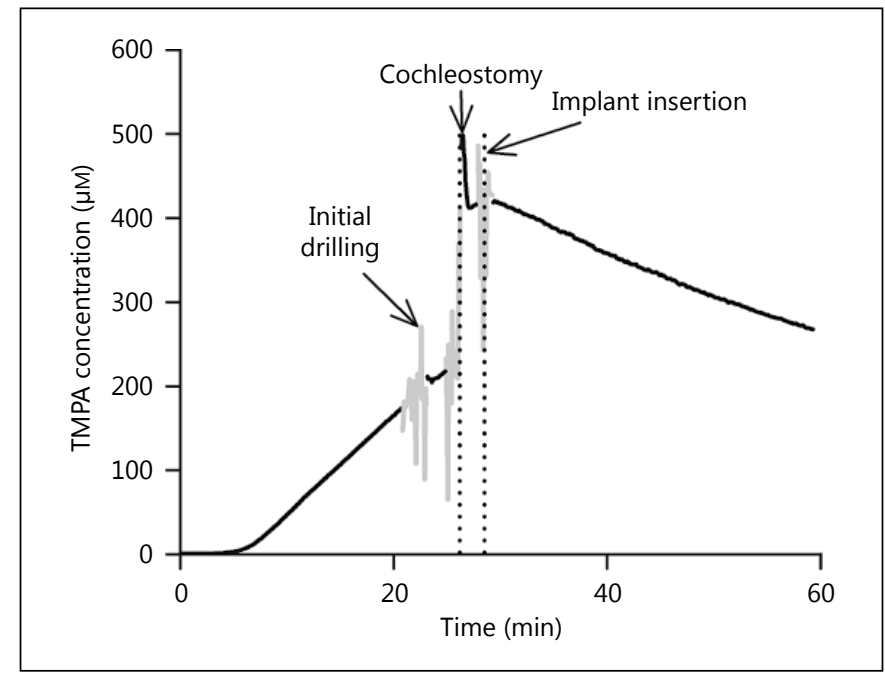

Fig. 2. TMPA time course recorded from the ST following RW irrigation with $20 \mathrm{mM}$ TMPA, followed by a drilled cochleostomy and implant insertion separated in time to allow periods of undisturbed TMPA recording. The initial (gray) noisy segment coincides with drilling the bone until perilymph was released, but with the perforation too small for the burr or the implant to enter the ST. The second gray segment shows the cochleostomy enlarged sufficiently for implant insertion. It was during this procedure that TMPA concentration increased substantially. Implant insertion had negligible immediate influence on TMPA concentration.

sive TMPA increase was not markedly disturbed. The fenestration was then enlarged sufficiently by drilling to permit implant insertion but pausing the procedure before the implant was inserted. At this time (fig. 2, cochleostomy) the TMPA concentration had increased substantially. Insertion of the implant (fig. 2, implant insertion) had only a minor influence on the measured TMPA concentration. This experiment clearly demonstrated that it was the act of drilling the cochleostomy, and specifically the tip of the rotating drill entering the fluid of ST, that caused the dramatic increase of concentration at the TMPA measurement site. The percentage increase of TMPA seen as a result of a drilled cochleostomy and implant insertion over 5 individual animals was 193.3, 156.8, 612.2, 406.3 and $191.1 \%$ (average $312.0 \%$, SD $174.2 \%)$.

In vitro studies confirmed that when a dental burr was activated in a narrow tube filled with simple electrolyte solution, it caused a localized intense stirring of the marker (fig. 3a). In contrast, an implant inserted into a closedended tube (allowing fluid efflux only at the insertion site) did not appreciably push fluorescent marker in front of it as it was inserted (fig. 3b).

Influence of Cochleostomy and Cochlear Implant Insertion on Drug Gradients
The entire procedures summarized in figure 3 are shown in the online supplementary video (for all online suppl. material, see www.karger.com/doi/10.1159/ 000353534), together with the same procedures shown in open solution (without nearby walls). The dental burr is shown to produce intense stirring and the movement of an implant through the solution is seen to drag nearby solution. In the latter experiment, the apparently viscous nature of the solution is the result of the magnification used and none of the solutions used had viscous properties that differed appreciably from perilymph.

Figure 4 shows a basal turn TMPA recording experiment in which the implantation was performed without the dental burr influencing perilymph. In this experiment, the bone at the cochleostomy site was thinned with a burr but the endosteum was opened with a fine pick similar to the approach used in soft surgery. This procedure was more time consuming but the combined fenestration and implant insertion has almost no influence on the measured TMPA concentration. The slow decline following implantation is again caused by TMPA washout by CSF at the base of the ST. The increase of TMPA seen as a result of a cochleostomy made with a pick followed by implant insertion averaged $13.2 \%($ SD $1.6 \%, \mathrm{n}=2)$.

TMPA or drug concentration changes as a result of implantation were most dramatic in the basal turn and were far less when measured at a site further from the implantation site. Figure 5 shows TMPA concentration measured in the second turn of the ST (estimated to be $7.5 \mathrm{~mm}$ from the base of the ST when measured along the scala) during a drilled cochleostomy and cochlear implant electrode insertion; procedures are comparable to those in figures 1 and 2 . In this experiment, implantation was performed at $60 \mathrm{~min}$ after TMPA irrigation started to allow a higher concentration to be established at the second turn recording site. Implantation only had a slight influence on the rising TMPA curve, seen as a transient slowing of the rate of increase. The results of this single experiment were consistent with calculated findings (below), and even smaller changes were expected with SS techniques, so the experiment was not repeated. The absence of large changes in the second turn is due to the larger distance and correspondingly prolonged diffusion times for changes in the basal turn to influence the second turn.

We used our computer model of the inner ear fluid spaces to assess whether the interpretation of TMPA changes during implantation was physically plausible. Figure 6 shows a detailed simulation of the experiments. This includes the following: (1) TMPA loading of the ST by entry at the RWM with a 2-mM RW niche con- 
Fig. 3. Influence of procedures demonstrated with fluorescent dye solution in 0.79-mm inner diameter glass capillaries filled with lactated Ringer's solution and part containing the solution with fluorescein. a Rotating dental burr inserted into the fluid. An entire segment is rapidly stirred and fluorescent dye distribution becomes homogenous within that segment. Regions beyond the stirred segment are unaffected. b Implant insertion into a closedend tube in which the only fluid outlet is at the insertion site. Dye solution is not pushed along the tube by the cochlear implant electrode tip but is dragged to some degree by the electrode body.
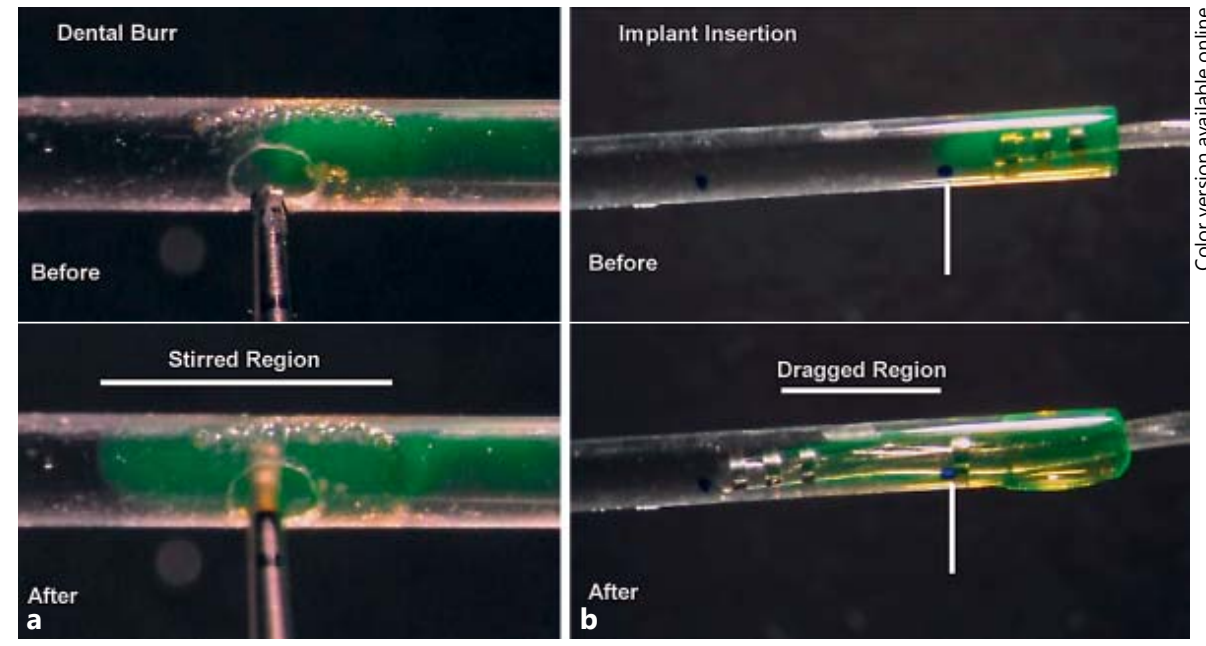

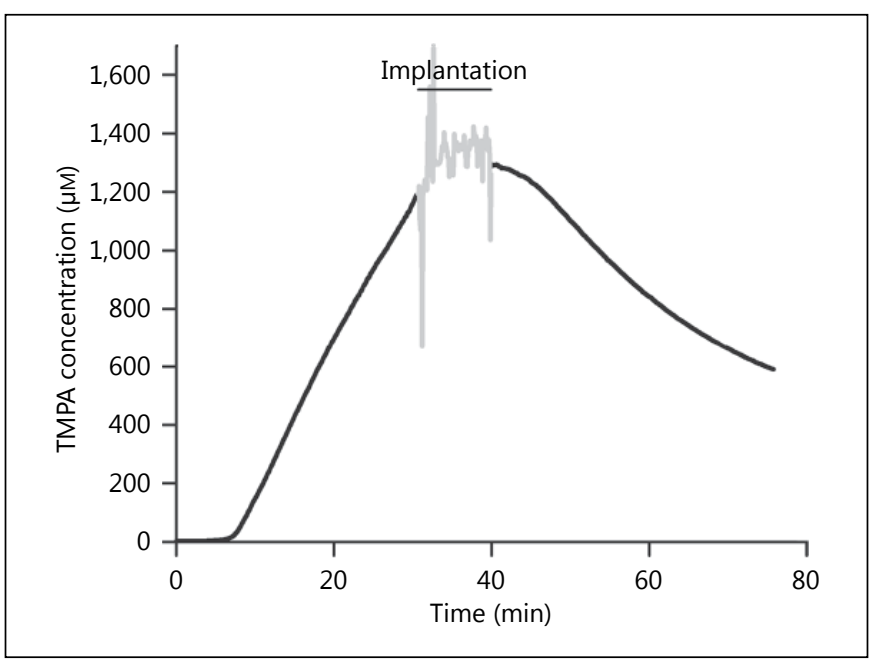

Fig. 4. TMPA time course recorded from the ST following RW irrigation with $20 \mathrm{mM}$ TMPA, followed by cochleostomy performed with SS techniques and implant insertion.

centration (RW permeability of $5 \times 10^{-7} \mathrm{~m} / \mathrm{s}$ ); (2) diffusion along the ST with a diffusion coefficient of $1.01 \times$ $10^{-9} \mathrm{~m}^{2} / \mathrm{s} \mathrm{combined} \mathrm{with} \mathrm{an} \mathrm{apically} \mathrm{directed} \mathrm{volume} \mathrm{flow}$ of $8 \mathrm{nl} / \mathrm{min}$ in the intact cochlea; (3) elimination from the ST to blood at $35 \mathrm{~min}$ half time, and (4) communication with adjacent compartments (spiral ligament, spiral ganglion - 10 min half time). At the time of cochleostomy, the stirring of perilymph by the dental burr was achieved by uniformly distributing the available TMPA over a short

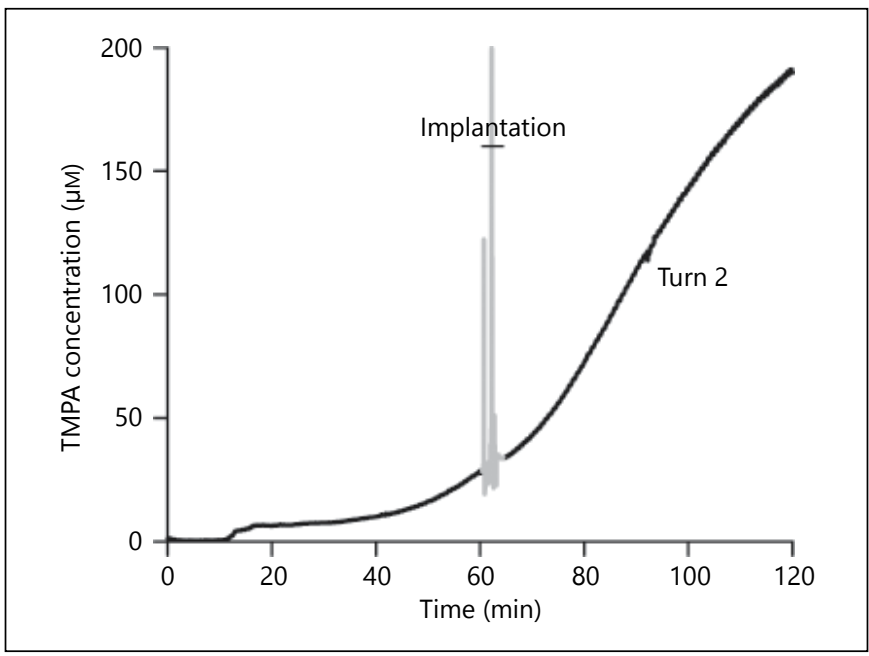

Fig. 5. TMPA time course recorded from the second turn of the ST following cochleostomy drilling. As it takes longer for TMPA to reach the second turn, the implantation procedure in this experiment was performed 60 min after RW irrigation commenced. Cochleostomy drilling and implantation which would induce changes in the basal turn had only minor influence on TMPA concentration measured in the second turn.

segment, as described below, followed by simulation of implant insertion for a $2.25-\mathrm{mm}$ distance over a 20 -second period. This included calculation of the displacement of perilymph from the ST at the cochleostomy site with appropriate volume flows and associated TMPA movements along the scala based on the implant and ST dimensions. Since cochlear perforation releases perilymph pressure, a volume flow simulating CSF entry at the CA and loss at the cochleostomy site at $200 \mathrm{nl} / \mathrm{min}$ replaced the 


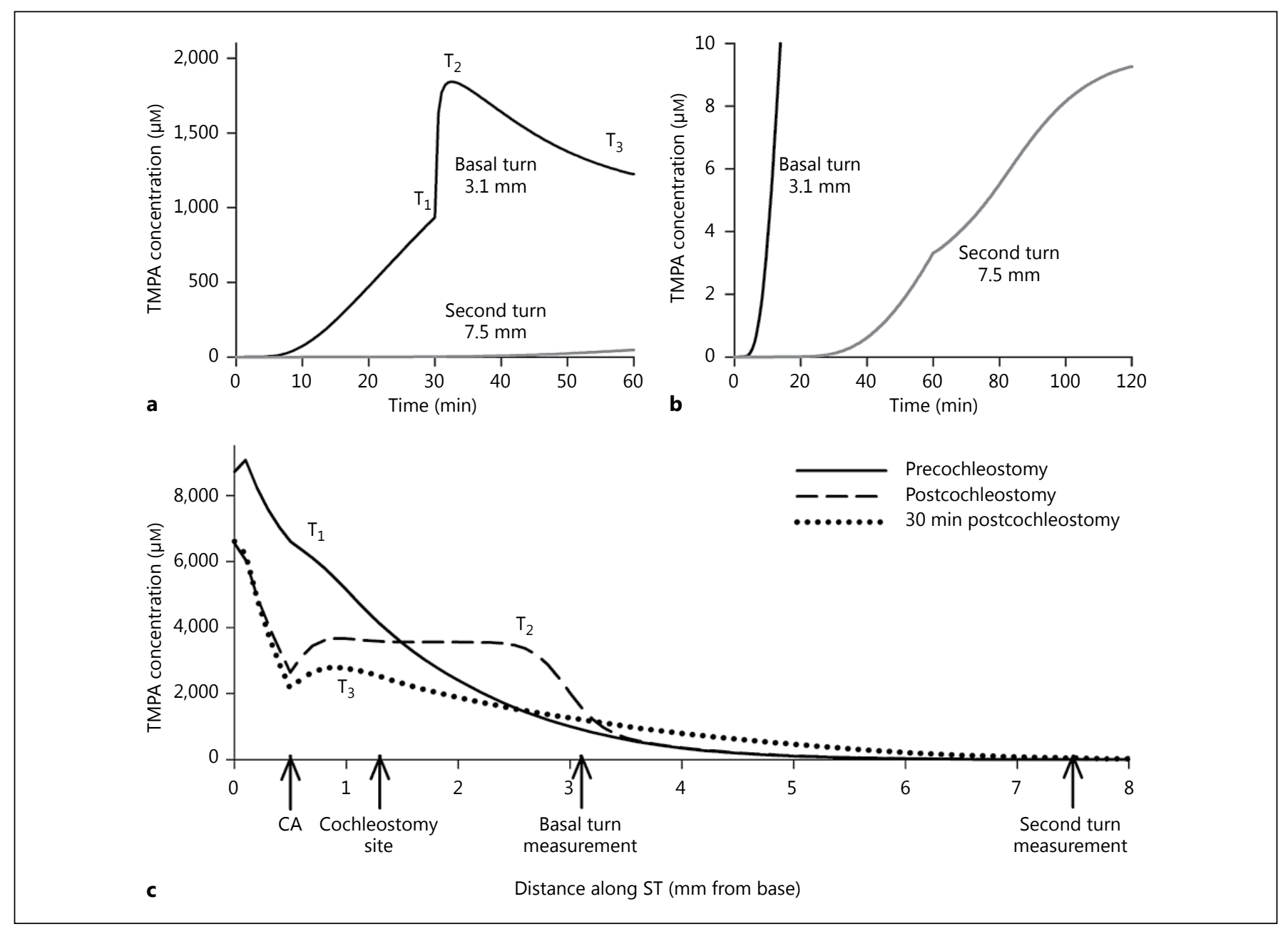

Fig. 6. TMPA time courses (a, b) and profile with distance along the ST (c) calculated with a program that simulates solute movements in the cochlear fluids. $\mathrm{T}_{1}, \mathrm{~T}_{2}$ and $\mathrm{T}_{3}$ show different time points in a for which the concentration profile with distance is shown in c. CA indicates the location in the model where the CA enters the ST. Prior to cochleostomy a substantial gradient for TMPA exists along the ST (trace $\mathrm{T}_{1}$ in $\mathbf{c}$ ). To simulate the fluids stirring resulting from a drilled cochleostomy, solute over the region up to $1.6 \mathrm{~mm}$ each side

apically directed volume flow at the time of cochleostomy. TMPA concentrations are shown as a function of time (fig. 6a, b) or with distance along the ST (fig. 6c). Figure 6 a shows the time course at the basal turn measurement site $(3.1 \mathrm{~mm}$ from the base) during the initial 30-min TMPA loading period. The TMPA gradient along the ST prior to implantation is shown as the curve for time $T_{1}$ in figure $6 \mathrm{c}$. Comparison of these curves shows that TMPA concentration at the basal end of the ST (near the RW) is predicted to be approximately 8 times higher than at the TMPA measurement site. It is this pool of high TMPA of the cochleostomy location was summed and distributed equally, equivalent to fully mixing the solute in the basal $2.9 \mathrm{~mm}$ from the ST. As the solution at the measurement site (at $3.1 \mathrm{~mm}$ from the base) becomes mixed with higher concentration (more basal solution), concentration measured at that site rapidly rises $(\mathbf{a})$. b Calculated time courses scaled to see the second turn measurement site and for a simulated cochleostomy occurring at $60 \mathrm{~min}$ after TMPA application commences (comparable to the protocol in fig. 5).

solution that allows the concentration at the measurement site to rise so rapidly during the cochleostomy. The stirring of ST contents during the cochleostomy drilling was implemented by dispersing the available TMPA (taking into account the varying volume with distance along the ST) evenly over a distance of $1.6 \mathrm{~mm}$ each side of the burr (a distance based on the in vitro experiments). This stirring accounts for the rapid increase (fig. $6 \mathrm{a}, \mathrm{T}_{2}$ ) and is reflected in the more even distribution with distance along the ST (fig. 6c, $\mathrm{T}_{2}$ ). Commencing at the time of cochleostomy, the simulation incorporates CSF entry at the aque- 
duct with flow towards and leakage at the cochleostomy site. This decreases concentration in the basal region and accounts for the subsequent decline of the time course (fig. 6a). The calculated concentration time course seen in the second turn during these events is also comparable with the measured curve (as shown in fig. 6b) for a 60-min pre-implantation loading period. This includes a slight slowing of the rate of increase seen at the time of implantation which may be accounted for by a slow rate of apical volume flow in the ST of the intact cochlea that ceases when the scala is perforated at the time of cochleostomy. In the calculations, an apical flow rate of $8 \mathrm{nl} / \mathrm{min}$ for the intact cochlea, which stopped at the time of cochleostomy, gives a small inflection of the curve (fig. 6b, second turn), which is more pronounced than that seen in the measured data. This analysis confirms that the measured TMPA time courses could arise from a stirring of the basal region of the ST during the cochleostomy procedure.

\section{Discussion}

This is the first study in which the alteration of perilymph marker distribution during cochleostomy and CI has been assessed by direct, real-time measurements of concentration. Our initial results showed a substantial rise in concentration at the measurement site in the upper basal turn of the ST at the time of implantation. This was first thought to be due to the cochlear implant electrode pushing a higher concentration solution from the region near the cochleostomy site apically to the recording site during insertion. More detailed experiments, however, revealed that this was not the case. The increase was found to be caused by the act of drilling the cochleostomy, with the perilymph near the cochleostomy site being stirred when a portion of the rotating burr entered the scala. The concentration rise caused by the cochleostomy procedure was immediately followed by a progressive decline in concentration. This was driven by the washout of TMPA-containing perilymph from the ST basal turn by CSF flow from the CA to the cochleostomy site and due to TMPA diffusing to regions of lower concentration. Conversely, the rapid concentration increase did not occur in the second turn of the ST, which is $6 \mathrm{~mm}$ from the cochleostomy site, when the cochleostomy was drilled, nor in the basal turn when SS techniques were used to make the cochleostomy. In vitro experiments confirmed that the marker was intensely stirred by a dental burr, creating complex flow and vortices, rapidly redistributing the marker a short dis- tance in both directions along the glass tube when the rotating dental burr came into contact with the fluid (fig. 3a). These data, therefore, show that implantation procedures can have a substantial and immediate influence on drug levels in the basal turn, which could in turn influence functional measurements if drug level plays a critical role in determining functional state. Functional measurements can also be influenced by other factors such as hearing loss associated with the surgical procedures.

Even though performing the cochleostomy by drilling resulted in a wider drug distribution along the ST than using SS techniques, which may be advantageous for expediting the delivery of protective drugs along the cochlea, drilling has several known disadvantages. These include the increased risk of acoustic trauma and the high likelihood of bone dust entering the ST, which may be a nidus for more extensive fibrosis and undesired osteoneogenesis. To avoid this, the 'ideal' cochleostomy is performed by 'blue-lining' the endosteum, which entails thinning the bone with the burr and then opening the endosteum with a pick, as was performed in some of these experiments. In practice, the endosteum is often breached and the waist of the drill can enter the scala as we have modeled here. We therefore conclude that drilling the cochleostomy is not advisable. However, inadvertent scalar entry with the drill may increase the reach of protective drugs if they are already present within the inner ear, which could be advantageous if this surgical event occurs.

Based on our observations from this study, we speculate that surgical drilling could potentially be used to widen the distribution of drug in other otological surgeries and, in particular, stapedotomy. Fenestration of the stapes footplate with a microdrill is a popular technique in stapedotomy, where the technique is analogous to that emulated here; many surgeons will introduce the burr into the stapedotomy to its waist in order to ensure consistency of the size of the fenestra. We have recently demonstrated the efficacy of stapedial drug delivery [King et al., 2011; Salt et al., 2012b], so if in the future this route were used to preload the vestibule prior to stapes surgery, drill-assisted stapedotomy would be expected to broaden the distribution of drug already present within the vestibular perilymph.

The act of inserting a cochlear implant electrode into the cochlea did not cause a rapid redistribution of intracochlear drug in the ST. The volume of perilymph displaced during electrode insertion into the basal turn of the ST is equivalent to the volume of the electrode. This causes fluid to be expelled from the cochleostomy and 
possibly through the $\mathrm{CA}$, carrying drug-laden perilymph in a basal direction since there is not an outlet at the apex. It was evident in the in vitro experiments that fluorescein was expelled from the open end of the tube during electrode insertion (in the opposite direction to the movement of the electrode), and the electrode tip passed through the fluorescein/Ringer's interface without pushing a bolus of fluorescein in front of it (fig. 3b). Conversely, when the closed end of the tube was perforated and the experiment repeated, fluorescein was pushed in front of the electrode tip during insertion due to the fluorescein being carried with the body of fluid leaking from the distal perforation (data not shown). These experiments demonstrate that bulk longitudinal flow is established toward the perforation site during electrode insertion, and the electrode tip does not push a bolus of drug in front of it when there is no distal perforation present.

Even though the bulk of the fluorescein was expelled from the open end of the tube during electrode insertion, we observed that a small amount was also being dragged along with the electrode array around its circumference (fig. 3b). This is presumably due to fluid viscosity and surface tension of fluids in small spaces. Other experiments (see online suppl. video) confirmed that the electrode drags fluorescein when the electrode is advanced back and forth through a stream of it. This may become an important consideration if the electrode is inserted, removed and reinserted during CI since presumably this would drag drug with it, altering intracochlear drug distribution.

The ramification of intracochlear drug being expelled with perilymph from the cochleostomy is that there may be lower levels of drug present in the basal turn than anticipated. However, it is expected that the local drug losses in the ST will be partially replenished by radial transfer from the scala vestibuli, due to the concentration gradient, when the vestibule is loaded with drug. Our previous studies [King et al., 2011; Salt et al., 2012b] show that in the guinea pig drug directly enters the oval window, probably through the annular ligament of the stapediovestibular joint, following intratympanic application. This can heavily load the vestibule with drug, making it act like a drug repository. This has also been shown in humans and rats [Zou et al., 2005].

Both the modeling and measurement in the second turn show that drug levels following local application to the RW niche are substantially lower in the second turn than in the basal turn. This is consistent with prior studies that have shown substantial longitudinal gradients of drug following local applications [Mynatt et al., 2006; Plontke et al., 2007; Plontke et al., 2008]. It is also consistent with Chang et al. [2009], who reported that longer waiting times between drug application and implantation achieved the best protection of function (optimal at $2 \mathrm{~h}$ ), assessed by ABR in guinea pigs. So even with the presence of drug gradients, therapeutic levels can be achieved in the second turn. There is greater concern if a primary goal of the drug therapy is to help preserve low-frequency (apical) sensitivity of the ear following implantation. As drug distribution is dominated by diffusion it may not be possible to achieve therapeutic drug levels in apical regions after short application times. In the human, drug levels reaching apical regions are likely to be even lower than the guinea pig as the ST is almost twice the length in the human. It is presently unknown whether drugs must reach the apex to protect low-frequency hearing or whether basal applications would be effective. If therapeutic drug levels at the apex are necessary, alternative strategies such as delivering drugs from the implant itself may be required.

The experimental data showed considerable variability in the absolute levels of TMPA found in perilymph following local application to the RW niche. This is due to the high variability of RWM permeability which has been reported in prior studies [Salt and Ma, 2001; Mynatt et al., 2006]. Intracochlear drug applications would overcome this source of variability.

This paper demonstrates that the method of performing the cochleostomy influences the distribution of drug present in the ST basal turn more than the insertion of the electrode array. We found that the distribution of drug in the ST basal turn can be expanded by the use of a surgical drill entering the scala. However, this is unlikely to assist with the preservation of residual hearing when steroids are used since the disturbance was not observed in the upper cochlear turns where the hair cells used for speech recognition reside. The stirring of perilymph could potentially be used to advantage in other clinical contexts such as stapedectomy, where a protective drug delivered to the oval window could be more widely distributed throughout the vestibule after stapedotomy.

\section{Acknowledgments}

The authors would like to thank Ruth Gill for preparing the figures and Dr. Tim Hullar for his suggested experiments. This study was supported by NIH/NIDCD research grant DC01368 and the National Health and Medical Research Council of Australia project grant 1007948 . 


\section{References}

Adunka O, Kiefer J, Unkelbach MH, Lehnert T, Gstoettner W: Development and evaluation of an improved cochlear implant electrode design for electric acoustic stimulation. Laryngoscope 2004;114:1237-1241.

Baumgartner WD, Jappel A, Morera C, Gstöttner W, Müller J, Kiefer J, Van De Heyning P, Anderson I, Nielsen SB: Outcomes in adults implanted with the FLEXsoft electrode. Acta Otolaryngol 2007;127:579-586.

Briggs RJ, Tykocinski M, Stidham K, Roberson JB: Cochleostomy site: implications for electrode placement and hearing preservation. Acta Otolaryngol 2005; 125:870-876.

Chang A, Eastwood H, Sly D, James D, Richardson R, O'Leary S: Factors influencing the efficacy of round window dexamethasone protection of residual hearing post-cochlear implant surgery. Hear Res 2009;255:67-72.

Cipolla MJ, Iyer P, Dome C, Welling DB, Bush ML: Modification and comparison of minimally invasive cochleostomy techniques: a pilot study. Laryngoscope 2012;122:1142-1147.

Coulson CJ, Taylor RP, Reid AP, Griffiths MV, Proops DW, Brett PN: An autonomous surgical robot for drilling a cochleostomy: preliminary porcine trial. Clin Otolaryngol 2008;33: 343-347.

Doménech J, Carulla M, Traserra J: Sensorineural high-frequency hearing loss after drill-generated acoustic trauma in tympanoplasty. Arch Otorhinolaryngol 1989;246:280-282.

Eastwood H, Pinder D, James D, Chang A, Galloway S, Richardson R, O'Leary S: Permanent and transient effects of locally delivered $n$ acetyl cysteine in a guinea pig model of cochlear implantation. Hear Res 2010;259:2430.

Enticott JC, Eastwood HT, Briggs RJ, Dowell RC, O'Leary SJ: Methylprednisolone applied directly to the round window reduces dizziness after cochlear implantation: a randomized clinical trial. Audiol Neurotol 2011;16:289_ 303.

Eshraghi AA: Prevention of cochlear implant electrode damage. Curr Opin Otolaryngol Head Neck Surg 2006;14:323-328.

Fishman AJ, Moreno LE, Rivera A, Richter CP: $\mathrm{CO}_{2}$ laser fiber soft cochleostomy: development of a technique using human temporal bones and a guinea pig model. Lasers Surg Med 2010;42:245-256.

Gantz BJ, Turner CW: Combining acoustic and electrical hearing. Laryngoscope 2003;113: 1726-1730.
Gantz BJ, Turner C: Combining acoustic and electrical speech processing: Iowa/Nucleus hybrid implant. Acta Otolaryngol 2004;124: 344-347.

Gantz BJ, Turner C, Gfeller KE, Lowder MW: Preservation of hearing in cochlear implant surgery: advantages of combined electrical and acoustical speech processing. Laryngoscope 2005; 115:796-802.

Gstoettner WK, van de Heyning P, O'Connor AF, Morera C, Sainz M, Vermeire K, Mcdonald S, Cavallé L, Helbig S, Valdecasas JG, Anderson I, Adunka OF: Electric acoustic stimulation of the auditory system: results of a multi-centre investigation. Acta Otolaryngol 2008;128: 968-975.

Gudis DA, Montes M, Bigelow DC, Ruckenstein MJ: The round window: is it the 'cochleostomy' of choice? Experience in 130 consecutive cochlear implants. Otol Neurotol 2012;33: 1497-1501.

James DP, Eastwood H, Richardson RT, O'Leary SJ: Effects of round window dexamethasone on residual hearing in a guinea pig model of cochlear implantation. Audiol Neurotol 2008; 13:86-96.

Jayawardena J, Kuthubutheen J, Rajan G: Hearing preservation and hearing improvement after reimplantation of pediatric and adult patients with partial deafness: a retrospective case series review. Otol Neurotol 2012;33:740-744.

Kiefer J, Gstoettner W, Baumgartner W, Pok SM, Tillein J, Ye Q, von Ilberg C: Conservation of low-frequency hearing in cochlear implantation. Acta Otolaryngol 2004;124:272-280.

Kiefer J, Ye Q, Tillein J, Adunka O, Arnold W, Gstöttner W: Protecting the cochlea during stapes surgery: is there a role for corticosteroids? Adv Otorhinolaryngol 2007;65:300307.

King EB, Salt AN, Eastwood HT, O'Leary SJ: Direct entry of gadolinium into the vestibule following intratympanic applications in guinea pigs and the influence of cochlear implantation. J Assoc Res Otolaryngol 2011;12:741751.

Kylén P, Arlinger S: Drill-generated noise levels in ear surgery. Acta Otolaryngol 1976;82: 402-409.

Lenarz T, Stover T, Buechner A, Paasche G, Briggs R, Risi F, Pesch J, Battmer RD: Temporal bone results and hearing preservation with a new straight electrode. Audiol Neurotol 2006; 11(suppl 1):34-41.

Lehnhardt E: Intracochlear placement of cochlear implant electrodes in soft surgery technique (in German). HNO 1993;41:356-359.
Mynatt R, Hale SA, Gill RM, Plontke SK, Salt AN: Demonstration of a longitudinal concentration gradient along scala tympani by sequential sampling of perilymph from the cochlear apex. J Assoc Res Otolaryngol 2006;7:182193.

Ohyama K, Salt AN, Thalmann R: Volume flow rate of perilymph in the guinea-pig cochlea. Hear Res 1988;35:119-129.

Pau HW, Just T, Bornitz M, Lasurashvilli N, Zahnert T: Noise exposure of the inner ear during drilling a cochleostomy for cochlear implantation. Laryngoscope 2007;117:535-540.

Plontke SK, Mynatt R, Gill RM, Salt AN: Concentration gradient along scala tympani following the local application of gentamicin to the round window membrane. Laryngoscope 2007;117:1191-1198.

Plontke SK, Biegner T, Kammerer B, Delabar U, Salt AN: Dexamethasone concentration gradients along scala tympani after application to the round window membrane. Otol Neurotol 2008:29:401-406.

Salt AN, Ma Y: Quantification of solute entry into cochlear perilymph through the round window membrane. Hear Res 2001;154:88-97.

Salt AN, Hartsock JJ, Gill RM, Piu F, Plontke SK: Perilymph pharmacokinetics of markers and dexamethasone applied and sampled at the lateral semi-circular canal. J Assoc Res Otolaryngol 2012a;13:771-783.

Salt AN, King EB, Hartsock JJ, Gill RM, O'Leary SJ: Marker entry into vestibular perilymph via the stapes following applications to the round window niche of guinea pigs. Hear Res 2012b;283:14-23.

Skarzynski H, Lorens A, Piotrowska A, Anderson I: Preservation of low frequency hearing in partial deafness cochlear implantation (PDCI) using the round window surgical approach. Acta Otolaryngol 2007;127:41-48.

von Ilberg C, Kiefer J, Tillein J, Pfenningdorff T, Hartmann R, Stürzebecher E, Klinke R: Electric-acoustic stimulation of the auditory system. New technology for severe hearing loss. ORL J Otorhinolaryngol Relat Spec 1999;61: 334-340.

Ye Q, Tillein J, Hartmann R, Gstoettner W, Kiefer J: Application of a corticosteroid (triamcinolone) protects inner ear function after surgical intervention. Ear Hear 2007;28:361-369.

Zou J, Pyykkö I, Bjelke B, Dastidar P, Toppila E: Communication between the perilymphatic scalae and spiral ligament visualized by in vivo MRI. Audiol Neurotol 2005a;10:145-152. 